\title{
Analysis of the Gyroscopic Stability of the Wheelset
}

\author{
Hao Dong, Jing Zeng, Liang Wu, and Huanyun Dai \\ Traction Power State Key Laboratory, Southwest Jiaotong University, Chengdu 0086 610031, China \\ Correspondence should be addressed to Hao Dong; fusiji@my.swjtu.edu.cn
}

Received 20 February 2014; Revised 9 May 2014; Accepted 15 May 2014; Published 2 June 2014

Academic Editor: Mickaël Lallart

Copyright (C) 2014 Hao Dong et al. This is an open access article distributed under the Creative Commons Attribution License, which permits unrestricted use, distribution, and reproduction in any medium, provided the original work is properly cited.

\begin{abstract}
The wheelset of the railway vehicle is a rotor which itself has gyroscopic effect. Nowadays, the rolling stock has entered the era of high speed, and the wheel rotates faster than in the past. The influence of gyroscopic effect on stability is little understood. Metelitsyn's inequality theorem for asymptotic stability has some advantages to analyze this problem although this method is sufficient but not necessary condition. Based on its deduction, the extremal eigenvalues criterion and compared with Routh-Hurwitz criterion, both are applied to solve the critical value of speed. Further, according to the instability criterion, gyroscopic contributory ratio is derived to study how the role the gyroscopic effect plays in stability. Moreover, the effect of gyroscopic matrix or gyroscopic terms pitch rotor inertia Iy on stability coefficient is investigated. The results show that Iy is a key factor to wheelset gyroscopic stability. The gyroscopic effect becomes significant, and the stability increases with increasing Iy. The results also indicate that the critical value of speed solved by Metelitsyn theorem is more conservative than the one it solved by Hurwitz criterion, which proves that Metelitsyn inequality theorem for asymptotic stability is a sufficient but not necessary condition in the way of attaining the numerical simulation result. Finally, the test for the influence of gyroscopic effect on stability needs to be further studied.
\end{abstract}

\section{Introduction}

Dynamic stability problem of railway vehicle has been studied since hunting motion phenomenon was discovered by Stephenson from the United Kingdom 100 years ago [1]. For security operation of railway vehicle, stability problem is of primary importance. Instability may increase the derailment risk and lead to disastrous consequences. Guan investigated derailment caused by instability [2]. Goodall and Iwnicki proposed a new method to assess the vehicle stability which mainly refers to the equivalent conicity between the wheel and rail contact [3]. Chen et al. investigated the stability of high speed trains passing curved rail in crosswind [4]. Zeng et al. presented the random energy theorem to investigate the phenomenon of train derail, which gives an effect way to reveal the principle of the world's problem "derailment" [5-7].

Hunting phenomenon mentioned before always exists in the railway vehicle running process because the lateral and yaw motions of the vehicle are coupled. Specifically, the unconverged hunting means instability. As the vehicle system is usually considered a nonlinear system, the critical speed corresponds to the point of limit-cycle oscillation disappearing and the linear critical speed corresponds to the Hopf bifurcation point (the more detailed analysis is given by [8]). Furthermore, the former is usually lower than the latter. In this case, it means that if the running speed is lower than the former one, the stationary motion is global stability. Otherwise, sometimes the former equals the latter and it means that the stationary motion will lose its stability if the operation speed is higher than the critical speed. True gives the whole process to determine the critical speed in [9].

Moreover, the wheelset is the key component of the whole vehicle. Many researchers have focused on the wheelset stability to clarify the mechanism of instability. Wickens investigated the nonlinear stability of railway wheelset and bogie with worn wheel treads [10]. He also deduced the analytic solution of the linear critical speed for a simple wheelset with primary suspension [11]. Knudsen et al. applied Poincare sections and Poincare maps to analyze the bifurcation and chaos phenomenon of a single wheelset [12]. Ahmadian and Yang used asymptotic method to investigate the hunting stability of a wheelset with primary suspension [13, 14]. Liu et al. studied the random stability of single wheelset with Zhu's stochastic bifurcation theorem [15]. Compared with previous 
study in deterministic systems, they yielded the critical speed range of wheelset in stochastic system and firstly found the first passage failure phenomenon of wheelset [16].

Many researchers solved the linear critical speed with using traditional Hurwitz criterion before. For example, Sedighi and Shirazi proposed equivalent functions method for complex nonlinearities to analyze the limit cycle of wheelset based on Yang and Ahmadian's asymptotic method, where the Hopf bifurcation point is solved also by Hurwitz criterion [17]. They also investigated the influence of parameters on linear critical speed [18]. He et al. used Hurwitz criterion and SQP algorithm to solve the nonlinear optimization stability problem of car [19]; see also the study by Law and Brand [20]. As for the nonlinear critical speed, it refers to the nonlinear bifurcation theory. Nevertheless, neither the nonlinear stability nor the traditional linear stability theorem is the concern of this paper. What we are concerned with is the gyroscopic effect analysis, and somehow the traditional linear stability theory is utilized for comparison.

Nowadays, we step into the age of high speed railway. China's high speed electric motor units (EMU) $\mathrm{CRH} 2$ and CRH3 run as fast as $300 \mathrm{~km} / \mathrm{h}$. Furthermore, in the near future, the operation speed of China's EMU CRH500 is possibly to get up to $500 \mathrm{~km} / \mathrm{h}$. With the operation speed increasing, the wheelset is a rotor, whose gyroscopic effect on stability must be further studied. In the study of rotor stability, Kirillov investigated the asymptotic stability domain of a linear autonomous nonconservative system with Lineard and Chipart criterion [21]. He found that the stability boundary was governed by Whitney umbrella singularity. Junfeng and Zhaolin investigated the stability of linear nonconservative systems subjected to potential, gyroscopic, and circulatory forces and Rayleigh damping with three stability theorems. They proved that the three theorems are convenient and useful [22]. Stephen C. Spry et al. discussed the stabilization of unstable vehicle, which is characterized by single wheel. Differing from railway vehicle of wheelset, the single wheel vehicle is stabilized only by gyroscopic effect [23]. In view of further development of the nonconservative stability theory of gyroscopic systems, Pommer and Kliem offer an overview of some of the recent researches on the stability and response bounds of linear system [24]. Kirillov analyzed the stability of a linear autonomous nonconservative system with an even number of DOF. In his study, an approximation of the boundary of the asymptotic stability domain near the singularity is explicitly found, and an analytical estimate of the critical gyroscopic parameter is obtained [25]. He also summarized a complete overview on the subject of nonconservative stability from the modern point of view [26].

Metelitsyn built his inequality theorem for asymptotic stability criterion $[27,28]$. Compared with Hurwitz criterion, his criterion is only sufficient but not necessary. Not only did Kliem and Seyranian [29] give a counter example to prove the defect of Metelitsyn's criterion, but also they developed Metelitsyn's criterion. Therefore, Metelitsyn's theorem has some advantages for clear physical meanings and mechanism in derivation process. What is more is that the basic principles of Metelitsyn theorem and Hurwitz criterion are somehow the same. Specifically, they are both inequality criteria and based on stationary motion (the details are presented in Section 2). As we mentioned above, Metelitsyn theorem is only sufficient but not necessary for inequality. This leads to the derived results differing from Hurwitz criterion as shown in Section 3. It is not the traditional linear critical speed that we focus on, but it is the value solved by Metelitsyn's criterion and the derived gyroscopic effect analysis that we investigate in this paper.

\section{Metelitsyn's Inequality Theorem for Asymptotic Stability}

Consider the following linear differential equation of the second order [22]:

$$
[\mathbf{M}] \ddot{x}+[\mathbf{D}+\mathbf{G}] \dot{x}+[\mathbf{P}+\mathbf{E}] x=0,
$$

where the dot denotes time differentiation, $x \in R^{m}$, and $[\mathbf{P}]$ and $[\mathbf{D}]$ are symmetric matrices. $[\mathbf{G}]$ and $[\mathbf{E}]$ are skewsymmetric matrices. These matrices $[\mathbf{P}]=[\mathbf{P}]^{T},[\mathbf{D}]=[\mathbf{D}]^{T}$, $[\mathbf{G}]=-[\mathbf{G}]^{T},[\mathbf{E}]=-[\mathbf{E}]^{T}$ are related to potential forces matrix, damping matrix, gyroscopic matrix, and circulant matrix [30].

The eigenvalue problem of (1) is

$$
\left(\lambda^{2}[\mathbf{M}]+\lambda[\mathbf{D}+\mathbf{G}]+[\mathbf{P}+\mathbf{E}]\right) u=0, \quad u \neq 0 .
$$

With normalized eigenvectors $u^{T} u=1$, we obtain the relation

$$
M \lambda^{2}+(D+i G) \lambda+(P+i E)=0
$$

where $M, D, P, G$, and $E$ are real quantities (known as Rayleigh quotients):

$$
\begin{aligned}
& M=u^{T}[M] u, \quad D=u^{T}[D] u, \\
& P=u^{T}[P] u, \quad i G=u^{T}[G] u, \\
& i E=u^{T}[E] u .
\end{aligned}
$$

Theorem 1. If linearized system (1) is asymptotic stable, all the eigenvalues of (2) have negative parts $\operatorname{Re} \lambda<0$ [28].

Besides, one of the sufficient and necessary conditions of all the eigenvalues $\operatorname{Re} \lambda<0$ is Hurwitz criterion. Meanwhile, Metelitsyn was the first to derive an inequality theorem for all the eigenvalues $\operatorname{Re} \lambda<0$. His theorem is depicted as follows $[25,26]$ :

$$
\begin{aligned}
& D>0, \\
& M E^{2}-G D E<D^{2} P .
\end{aligned}
$$

Compared with Hurwitz criterion, Metelitsyn theorem is a sufficient but not necessary condition. But its matrices terms have physical meanings. This theorem provides a way to study the influence of gyroscopic effect on stability. 


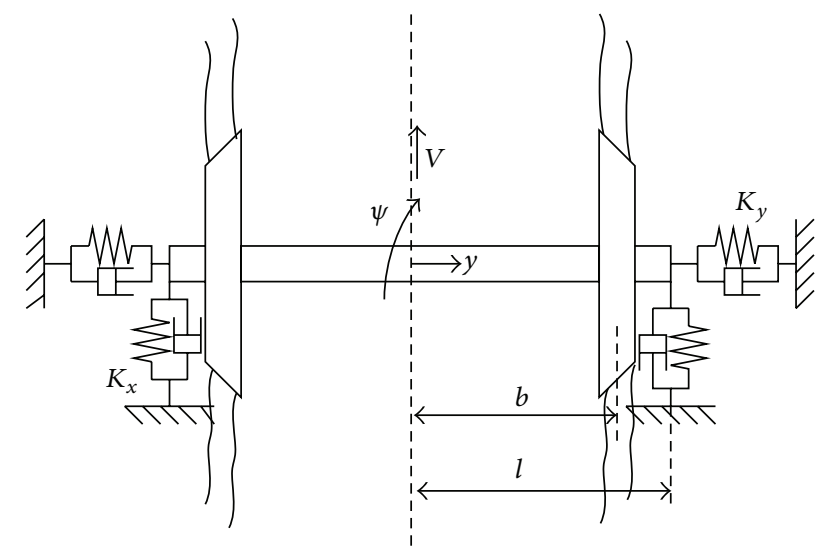

FIGURE 1: Dynamic model of wheelset with primary suspension.

Moreover, based on Lancaster and Tismenetsky's theorem [31], the corresponding quantities $M, D, P$ (Rayleigh quotients) are therefore limited by their eigenvalues:

$$
\begin{gathered}
M_{\text {min }}=\lambda_{\min }([\mathbf{M}]) \leq M \leq \lambda_{\max }([\mathbf{M}])=M_{\max }, \\
D_{\text {min }}=\lambda_{\text {min }}([\mathbf{D}]) \leq D \leq \lambda_{\max }([\mathbf{D}])=D_{\max }, \\
P_{\min }=\lambda_{\min }([\mathbf{P}]) \leq P \leq \lambda_{\max }([\mathbf{P}])=P_{\max },
\end{gathered}
$$

with

$$
G_{\max }=\lambda_{\text {max }}(i[\mathbf{G}]), \quad E_{\max }=\lambda_{\text {max }}(i[\mathbf{E}]) .
$$

So we have

$$
\begin{array}{cl}
-G_{\max } \leq G \leq G_{\max }, & -E_{\max } \leq E \leq E_{\max }, \\
G_{\min }=-G_{\max }, & E_{\min }=-E_{\max } .
\end{array}
$$

Therefore, inequality (5) yields a more sufficient stability condition:

$$
D_{\min }\left(D_{\min } P_{\min }+G_{\min } E_{\min }\right)-M_{\max } E_{\max }^{2}>0 .
$$

Compared with inequality (5), inequality (10) no longer depends on the eigenvectors $u$. This makes it suitable for engineering applications.

\section{Wheelset Lateral Equation}

Consider a wheelset model which contains the primary suspension. Its dynamic model is depicted in Figure 1. And (9) presents its lateral motion [13,32]. Consider

$$
\begin{gathered}
\frac{M}{\left[\begin{array}{cc}
m & 0 \\
0 & J
\end{array}\right]}\left[\begin{array}{l}
\ddot{y} \\
\ddot{\varphi}
\end{array}\right]+\left[\begin{array}{cc}
\frac{2 f_{22}}{V} & \frac{2 f_{23}}{V}-\frac{I_{y} V \lambda}{r_{0} b} \\
\frac{I_{y} V \lambda}{r_{0} b}-\frac{2 f_{23}}{V} & \frac{2 f_{11} l^{2}}{V}
\end{array}\right]\left[\begin{array}{c}
\dot{y} \\
\dot{\varphi}
\end{array}\right] \\
+\left[\begin{array}{cc}
2 k_{y}+\frac{W \lambda}{b} & -2 f_{22} \\
\frac{2 f_{11} b \lambda}{r_{0}} & 2 l_{0}^{2} k_{x}-W \lambda b+2 f_{23}
\end{array}\right]\left[\begin{array}{l}
y \\
\varphi
\end{array}\right]=0 .
\end{gathered}
$$

Therefore,

$$
\begin{aligned}
& {[\mathbf{D}]=\frac{\left[\mathbf{C}+\mathbf{C}^{\mathbf{T}}\right]}{2}=\left[\begin{array}{cc}
\frac{2 f_{22}}{V} & 0 \\
0 & \frac{2 f_{11} l^{2}}{V}
\end{array}\right],} \\
& {[\mathbf{G}]=\frac{\left[\mathbf{C}-\mathbf{C}^{\mathbf{T}}\right]}{2}=\left[\begin{array}{cc}
0 & \frac{2 f_{23}}{V}-\frac{I_{y} V \lambda}{r_{0} b} \\
I_{y} V \lambda & 2 f_{23} \\
r_{0} b & 0
\end{array}\right],} \\
& {[\mathbf{P}]=\frac{\left[\mathbf{K}+\mathbf{K}^{\mathbf{T}}\right]}{2}=\left[\begin{array}{cc}
2 k_{y}+\frac{W \lambda}{b} & \frac{f_{11} \lambda b}{r_{0}}-f_{22} \\
\frac{f_{11} \lambda b}{r_{0}}-f_{22} & 2 l_{0}^{2} k_{x}-W \lambda b+2 f_{23}
\end{array}\right],} \\
& {[\mathbf{E}]=\frac{\left[\mathbf{K}-\mathbf{K}^{\mathbf{T}}\right]}{2}=\left[\begin{array}{cc}
0 & -f_{22}-\frac{f_{11} \lambda b}{r_{0}} \\
f_{22}+\frac{f_{11} \lambda b}{r_{0}} & 0
\end{array}\right] .}
\end{aligned}
$$

\section{Asymptotic Domain of Railway Wheelset by Metelitsyn's Theorem}

Firstly, we yield the limited eigenvalues of the matrices $M, D$, $P$. With parameters in Table 1, we have

$$
\begin{gathered}
M_{\text {min }}=\lambda_{\text {min }}([\mathbf{M}])=J, \quad M_{\max }=\lambda_{\text {max }}([\mathbf{M}])=m, \\
D_{\text {min }}=\lambda_{\text {min }}([\mathbf{D}])=D_{\text {max }}=\lambda_{\text {max }}([\mathbf{D}])=\frac{2 f_{22}}{V}=\frac{2 f_{11} l^{2}}{V}, \\
G_{\text {max }}=\lambda_{\text {max }}(i[\mathbf{G}])=\left|\frac{2 f_{23}}{V}-\frac{I_{y} V \lambda}{r_{0} b}\right|, \\
E_{\text {max }}=\lambda_{\text {max }}(i[\mathbf{E}])=f_{22}+\frac{f_{11} \lambda b}{r_{0}}, \\
P_{\text {min }}=\lambda_{\text {min }}([\mathbf{P}])=\frac{A+\sqrt{A^{2}+4 b r_{0}^{2} B}}{-2 b r_{0}^{2}}, \\
P_{\max _{1}}=\lambda_{\text {max }}([\mathbf{P}])=\frac{A-\sqrt{A^{2}+4 b r_{0}^{2} B}}{-2 b r_{0}^{2}},
\end{gathered}
$$

where

$$
\begin{aligned}
A=- & 2 b f_{23} r_{0}^{2}-2 b k_{y} r_{0}^{2}-2 b k_{x} l^{2} r_{0}^{2}-\lambda r_{0}^{2} W+b^{2} \lambda r_{0}^{2} W, \\
B= & b^{2} f_{11}^{2} \lambda^{2}-2 b^{2} f_{11} f_{22} \lambda r_{0}+b f_{22}^{2} r_{0}^{2}-4 b f_{23} k_{y} r_{0}^{2} \\
& -4 b k_{x} k_{y} l^{2} r_{0}^{2}-2 f_{23} \lambda r_{0}^{2} W+2 b^{2} k_{y} \lambda r_{0}^{2} W \\
& -2 k_{x} l^{2} \lambda r_{0}^{2} W+b \lambda^{2} r_{0}^{2} W^{2} .
\end{aligned}
$$


Substituting (14) into inequality (10) yields

$$
\begin{aligned}
& \frac{2 f_{22}}{V}\left(\frac{2 f_{22}}{V} \times \frac{A+\sqrt{A^{2}+4 b r_{0}^{2} B}}{-2 b r_{0}^{2}}\right. \\
& \left.+\left|\frac{2 f_{23}}{V}-\frac{I_{y} V \lambda}{r_{0} b}\right|\left(f_{22}+\frac{f_{11} \lambda b}{r_{0}}\right)\right) \\
& -m\left(f_{22}+\frac{f_{11} \lambda b}{r_{0}}\right)^{2}>0 \\
& \Longrightarrow V<\left(\left(-2 A f_{22}^{2}-4 b^{2} f_{11} f_{22} f_{23} \lambda r_{0}-4 b f_{22}^{2} f_{23} r_{0}^{2}\right.\right. \\
& \left.-2 f_{22}^{2} \sqrt{A^{2}+4 b B r^{2}}\right) \\
& \times\left(-2 b f_{11} f_{22} I_{y} \lambda^{2}+b^{3} f_{11}^{2} \lambda^{2} m\right. \\
& -2 f_{22}^{2} I_{y} \lambda r_{0}+2 b^{2} f_{11} f_{22} \lambda m r_{0} \\
& \left.\left.+b f_{22}^{2} m r^{2}\right)^{-1}\right)^{1 / 2} \\
& \Longrightarrow V_{C M}=\left(\left(-2 A f_{22}^{2}-4 b^{2} f_{11} f_{22} f_{23} \lambda r_{0}-4 b f_{22}^{2} f_{23} r_{0}^{2}\right.\right. \\
& \left.-2 f_{22}^{2} \sqrt{A^{2}+4 b B r^{2}}\right) \\
& \times\left(-2 b f_{11} f_{22} I_{y} \lambda^{2}+b^{3} f_{11}^{2} \lambda^{2} m\right. \\
& -2 f_{22}^{2} I_{y} \lambda r_{0}+2 b^{2} f_{11} f_{22} \lambda m r_{0} \\
& \left.\left.+b f_{22}^{2} m r^{2}\right)^{-1}\right)^{1 / 2}
\end{aligned}
$$

where $V_{C M}$ represents the critical value of $V$ solved by Metelitsyn theorem.

We can solve the linear critical speed according to (16) and Hurwitz criterion [17-20,33], hereinafter referred to as $V_{C M}$ and $V_{\mathrm{cr}}$. With parameters in Table 1, it yields

$$
V_{C M}=88.36 \mathrm{~m} / \mathrm{s}, \quad V_{\mathrm{cr}}=126.38 \mathrm{~m} / \mathrm{s} .
$$

As we know, Hurwitz criterion is the sufficient and necessary condition for all eigenvalues having negative part and the result $V_{C M}<V_{\text {cr }}$ proves that Metelitsyn theorem is the sufficient but not necessary condition for asymptotic stability. Besides, based on equality (16) and Hurwitz criterion, we can solve the influence of parameters on $V_{C M}$ and $V_{\mathrm{cr}}$ as shown in Figures 2-4.

From Figures 2 and 3, we can also find $V_{C M}<V_{\mathrm{cr}}$. It has been again proved that Metelitsyn theorem is the sufficient but not necessary condition for asymptotic stability. Therefore, when this theorem is used for analysis, the corresponding $V_{C M}$ is safe but conservative.
TABLE 1: Parameters of wheelset.

\begin{tabular}{lcl}
\hline Parameters & Value & Commet \\
\hline$m$ & $1500 \mathrm{~kg}$ & Wheelset mass \\
$J$ & $825.7 \mathrm{~kg} \cdot \mathrm{m}^{2}$ & Yaw inertia \\
$f_{11}$ & $6.728 \mathrm{MN}$ & Longitudinal creep coefficient \\
$f_{22}$ & $6.728 \mathrm{MN}$ & Lateral creep coefficient \\
$f_{23}$ & $1500 \mathrm{~N} \cdot \mathrm{m}$ & Spin creep coefficient \\
$I_{y}$ & $70 \mathrm{~kg} \cdot \mathrm{m}^{2}$ & Pitch rotor inertia \\
$\lambda$ & 0.28 & Equivalent conicity \\
$r_{0}$ & $0.533 \mathrm{~m}$ & Wheel radius \\
$b$ & $0.7175 \mathrm{~m}$ & Half of rolling cycle gauge \\
$l_{0}$ & $1 \mathrm{~m}$ & Half of primary suspension gauge \\
$k_{x}$ & $5 \mathrm{MN} / \mathrm{m}$ & Primary longitudinal stiffness \\
$k_{y}$ & $5 \mathrm{MN} / \mathrm{m}$ & Primary lateral stiffness \\
$W$ & $\mathrm{~m} * \mathrm{~g}$ & Axle load \\
$V$ & - & Operation speed \\
\hline
\end{tabular}

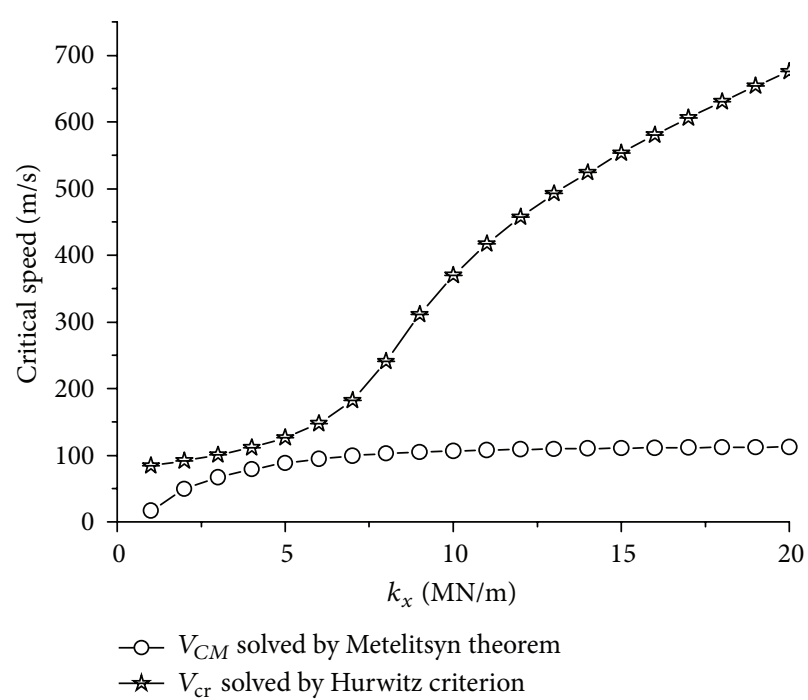

FIgURE 2: Influence of $k_{x}$ on critical value $V$.

\section{Gyroscopic Effect on Wheelset Stability}

5.1. Gyroscopic Contributory Ratio. As mentioned in Section 1, the single wheel vehicle is stabilized only by gyroscopic effect [12]. But it is the railway wheelset that we discuss in this paper, with primary suspension, whose main factors for stabilization are the primary suspension. However, how is the gyroscopic effect for stabilization of railway wheelset represented?

To represent the gyroscopic effect, from inequality (8), we can define stability coefficient $a_{t}$ with gyroscopic term and $a_{n t}$ without gyroscopic term:

$$
a_{t}=\frac{D_{\min }\left(D_{\min } P_{\min }+G_{\min } E_{\min }\right)}{M_{\max } E_{\max }^{2}}, \quad a_{n t}=\frac{D_{\min }^{2} P_{\min }}{M_{\max } E_{\max }^{2}} .
$$

Thus, the sufficient condition for asymptotical stability is $a_{t}>$ 1 or $a_{n t}>1$. 


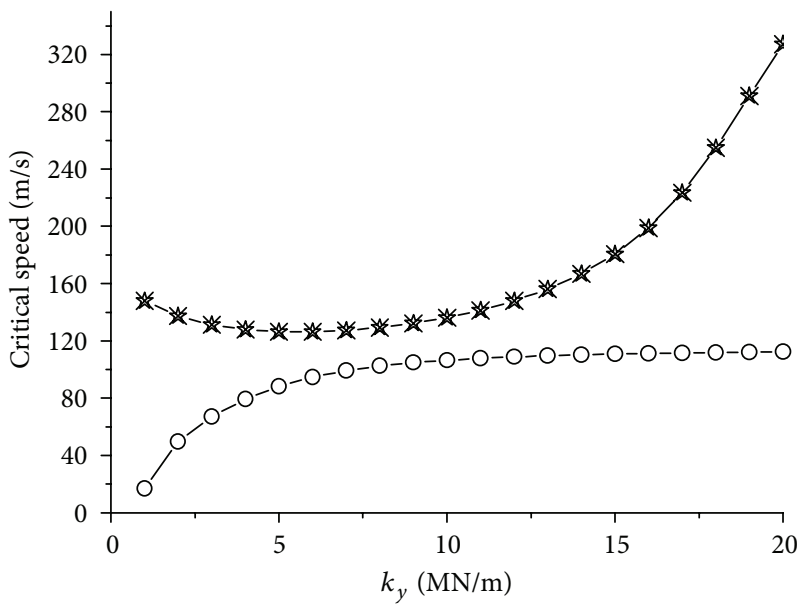

-O- $V_{C M}$ solved by Metelitsyn theorem

* $-V_{\text {cr }}$ solved by Hurwitz criterion

Figure 3: Influence of $k_{y}$ on critical value $V$.

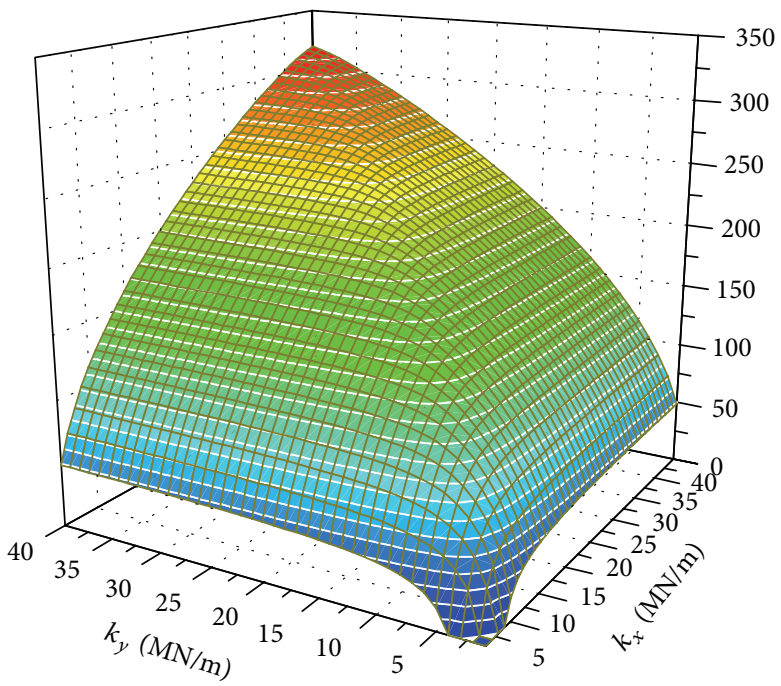

FIGURE 4: Influence of $k_{x}$ and $k_{y}$ on $V_{C M}$.

Furthermore, gyroscopic contributory ratio (GCR) can be defined as

$$
\mathrm{GOR}=\frac{a_{t}-a_{n t}}{a_{t}}=\frac{G_{\max } E_{\max }}{D_{\min } K_{\min }+G_{\min } E_{\min }} .
$$

With wheelset parameters, we can obtain the following.

It can be seen in Figure 5 that, with the equivalent conicity increasing, the gyroscopic contributory ratio for stabilization increases. As we know, the larger equivalent conicity leads to the worse stability. Meanwhile, the gyroscopic effect on stability becomes more obvious.

Next, we continue to discuss the gyroscopic effect on stability under $V=300 \mathrm{~km} / \mathrm{h}(83 \mathrm{~m} / \mathrm{s})$ of high speed railway operation speed and $V_{C M}$ condition. The influences of positioning stiffness $k_{x}$ or $k_{y}$ on $V_{C M}$ and on GOR for both $V_{C M}$ and high speed railway operation speed $V=300 \mathrm{~km} / \mathrm{h}$ are presented in Figures 6-7.

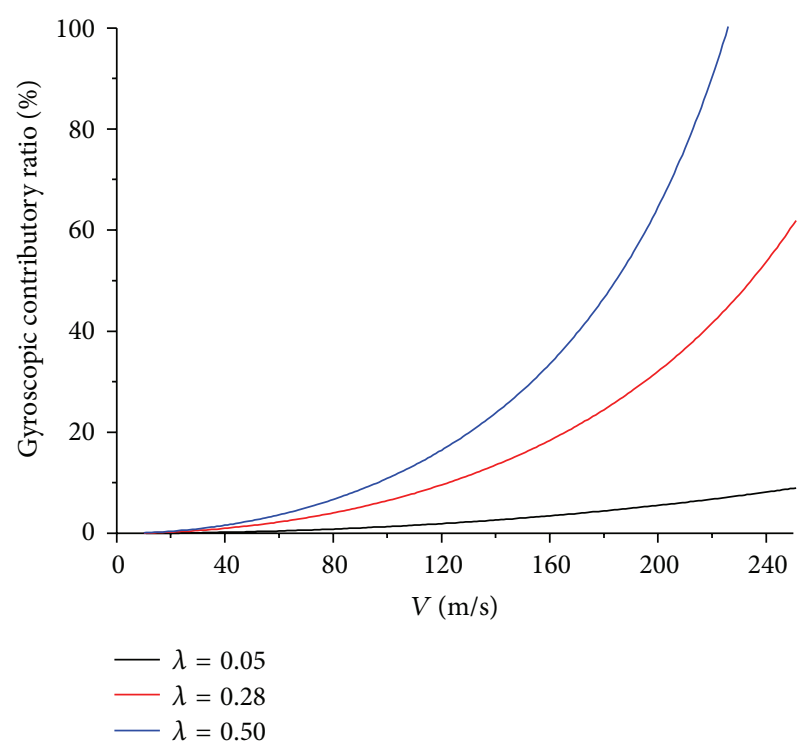

FIGURE 5: Gyroscopic contributory ratio.

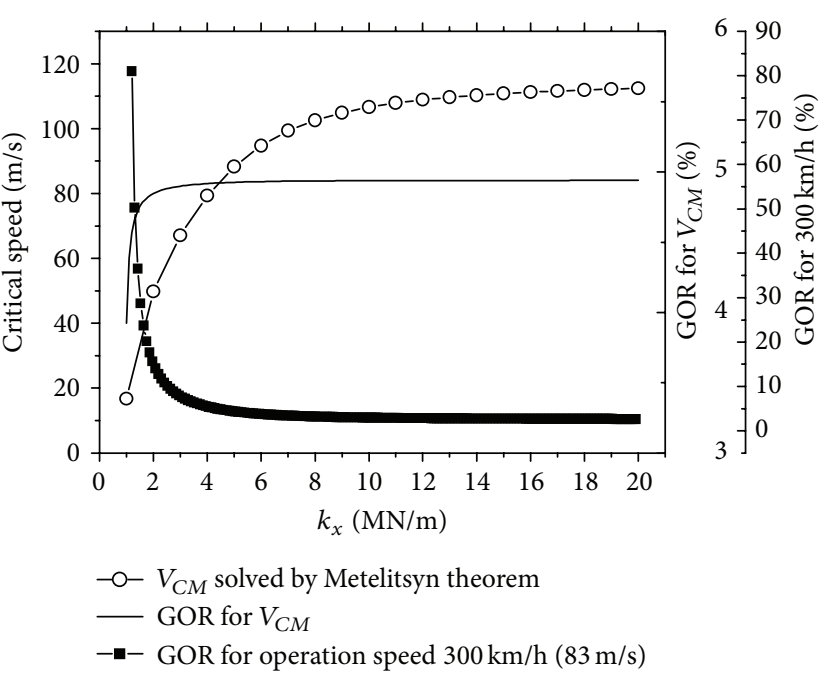

Figure 6: Influence of $k_{x}$ on $V_{C M}$ and GOR.

As shown in Figures 6 and 7, the results show that GOR for $V_{C M}$ increases slightly along with increasing $k_{x}$, and, further, with $k_{x}$ increasing, both GOR for $V_{C M}$ and $V_{C M}$ itself tend to be levelled off. Hence, overall, the change of GOR for $V_{C M}$ is small, and the value of GOR for $V_{C M}$ is as low as less than $5 \%$. If, however, GOR for high operation speed $300 \mathrm{~km} / \mathrm{h}$ firstly decreases a lot along with increasing $k_{x}$ and further with $k_{x}$ increasing, it also tends to be levelled off. That is, GOR for high operation speed $300 \mathrm{~km} / \mathrm{h}$ drops significantly from $80 \%$ to less than $5 \%$. This phenomenon indicates that GOR is insignificant when the operation speed is low and even if it gets to $V_{C M}$. But when $V_{C M}$ is low and the operation speed gets much higher than $V_{C M}$, GOR will become very significant. 


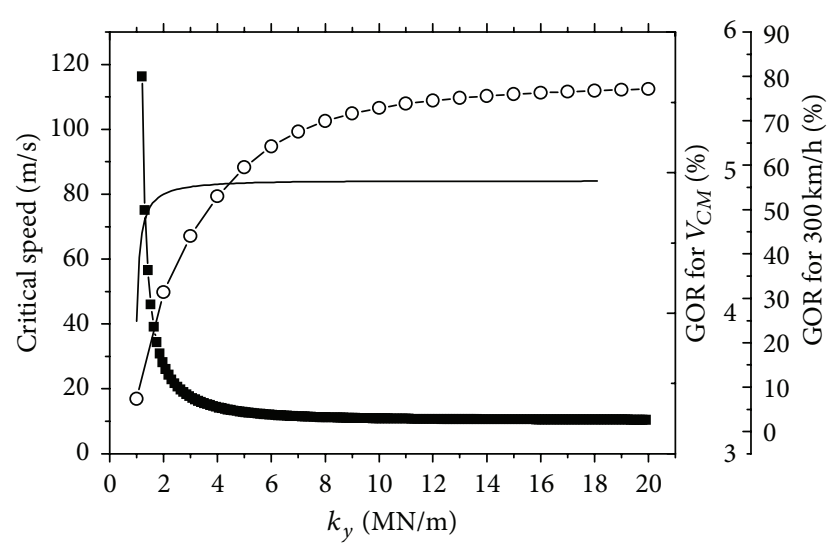

$$
\begin{aligned}
& \text { - } V_{C M} \text { solved by Metelitsyn theorem } \\
& \text { - GOR for } V_{C M} \\
& -- \text { GOR for operation speed } 300 \mathrm{~km} / \mathrm{h}(83 \mathrm{~m} / \mathrm{s})
\end{aligned}
$$

FIGURE 7: Influence of $k_{y}$ on $V_{C M}$ and GOR.

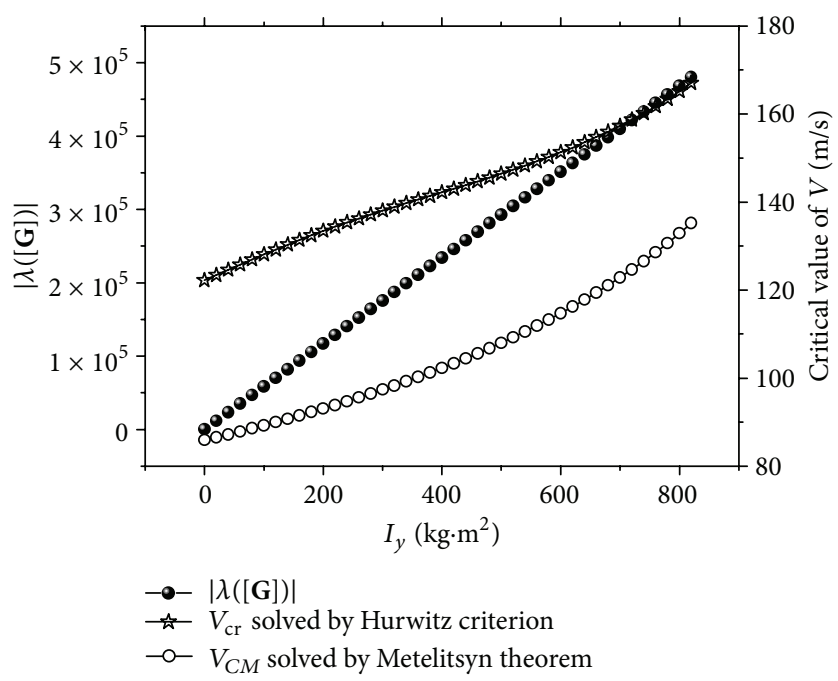

FIGURE 8: Influence of $I_{y}$ on $|\lambda([\mathbf{G}])|$ and critical value of $V$.

5.2. The Influence of Gyroscopic Term on Stability. We reconsider the gyroscopic matrix

$$
[\mathbf{G}]=\left[\begin{array}{cc}
0 & \frac{2 f_{23}}{V}-\frac{I_{y} V \lambda}{r_{0} b} \\
\frac{I_{y} V \lambda}{r_{0} b}-\frac{2 f_{23}}{V} & 0
\end{array}\right] .
$$

It contains the terms $f_{23}, I_{y}, r_{0}, b, \lambda$. Furthermore, among the above five terms, we can find that the pitch rotor inertia $I_{y}$ is the only factor which just affects [G] but does not affect the other matrices. For this reason, the influence of univariate $I_{y}$, which means the gyroscopic effect, on stability needs to be studied. The influence of $I_{y}$ on $\lambda([\mathbf{G}])$ and critical value of $V$ are shown in Figure 9.

In Figures 8 and 9, the results indicate that, with increasing $I_{y}$, the critical values of $V_{C M}$ and $V_{\mathrm{cr}}$ both increase but $V_{C M}$ is lower than $V_{\text {cr }}$. This again proves that the result attained

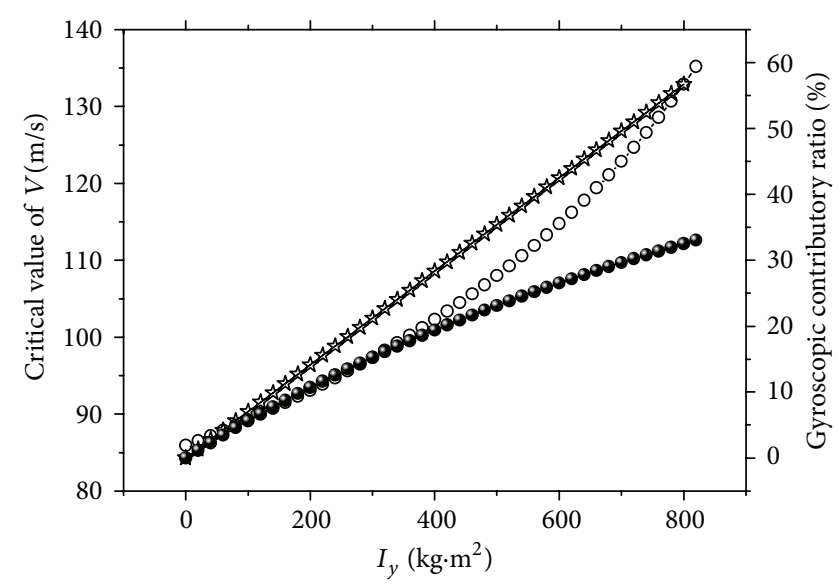

$$
\begin{aligned}
\text {-o- } & V_{C M} \text { solved by Metelitsyn theorem } \\
\text { - } & \text { Gyroscopic contributory ratio in } V_{C M} \text { condition } \\
\rightarrow- & \text { Gyroscopic contributory ratio in operation speed } \\
& 300 \mathrm{~km} / \mathrm{h}(83 \mathrm{~m} / \mathrm{s}) \text { condition }
\end{aligned}
$$

FIgURE 9: Influence of $I_{y}$ on $V_{C M}$ and GOR.

by Metelitsyn theorem is more conservative than the one attained by Hurwitz criterion. And further, with increasing $I_{y},|\lambda([\mathbf{G}])|$ and GOR also increase. This illustrates that $I_{y}$ is a key factor in wheelset gyroscopic stability. If it is greater, the gyroscopic effect becomes more significant and $V_{C M}$ gets higher.

\section{Conclusion and Outlook}

We derive the critical value of $V$ and gyroscopic contributory ratio of wheelset equation with Metelitsyn inequality theorem. Analytical and numerical result shows that pitch rotor inertia $I_{y}$ plays a key role in gyroscopic matrix. The gyroscopic effect becomes significant, and the $V_{C M}$ gets higher with increasing $I_{y}$. However, when $V_{C M}$ is low, but with high operation speed, gyroscopic contributory ratio is large. Therefore, to ensure high $V_{C M}$ of vehicle as well, increasing pitch rotor inertia can further increase the stability. In practice, to get larger value of pitch rotor inertia, one way is to increase the mass of the wheelset, and another way is to increase the radius of the wheelset, both of which can get higher linear critical speed as generally realized.

What is mentioned above just refers to the theoretical analysis. As we know, the pitch rotor inertia $I_{y}$ is the main factor in the gyroscopic system, and it would provide a method for testing verification. In the past EMU test on roller rig [34], we mainly focused on the influence of suspension parameters such as $k_{x}$ and $k_{y}$ on stability but ignored $I_{y}$. Nowadays, in the era of high speed EMU development, therefore, the new test item "gyroscopic effect on stability" is further required. Since China's roller rig has the condition of software and hardware, the following work of how to test the influence of gyroscopic effect on stability needs to be conducted. 


\section{Conflict of Interests}

The authors declare that there is no conflict of interests regarding the publication of this paper.

\section{Acknowledgments}

This work was supported by the National Basic Research Program of China ("973" Program) (Grant no. 2011CB711106), the National High Technology Research and Development Program of China ("863" Program) (Grant no. 2012AA112001), the National Key Technology Support Program of China (Grant no. 2009BAG12A01), the Southwest Jiaotong University 1st Outstanding Innovative Talents Fund, and the Doctoral Student Innovation Fund of Southwest Jiaotong University.

\section{References}

[1] D. C. F. Marshall, A History of British Railways down to the Year 1830, Oxford University Press, London, UK, 1938.

[2] Q. H. Guan, Study on the derailment mechanism and running safety of trains [Ph.D. thesis], Southwest Jiaotong University, Chengdu, China, 2010 (Chinese).

[3] R. Goodall and S. Iwnicki, "Non-linear dynamic techniques v. equivalent conicity methods for rail vehicle stability assessment," Vehicle System Dynamics, vol. 41, pp. 517-527, 2001.

[4] R. L. Chen, Q. Y. Zeng, X. G. Zhong, J. Xiang, X. G. Guo, and G. Zhao, "Numerical study on the restriction speed of train passing curved rail in cross wind," Science in China E: Technological Sciences, vol. 52, no. 7, pp. 2037-2047, 2009.

[5] Q. Y. Zeng, J. Xiang, Z. H. Zhou, and P. Lou, Analysis of Theory and Application of Train Derailment, Central South University Press, Changsha, China, 2006 (Chinese).

[6] J. Xiang, Q. Y. Zeng, and Z. H. Zhou, "Mechanical mechanism and random energy analysis theory of train derailment on the bridge and its application," Journal of The China Railway Society, vol. 26, no. 2, pp. 97-104, 2004 (Chinese).

[7] Q. Y. Zeng, J. Xiang, and P. A. Lou, "Breakthrough in solving the problem of train derailment - the approach of random energy analysis," Engineering Science, vol. 4, no. 12, pp. 9-20, 2002 (Chinese).

[8] H. True, "Does a critical speed for railroad vehicles exist?" in Proceedings of the 1994 ASME/IEEE Joint Railroad Conference, pp. 125-131, March 1994.

[9] H. True, "On the theory of nonlinear dynamics and its applications in vehicle systems dynamics," Vehicle System Dynamics, vol. 31, no. 5-6, pp. 393-421, 1999.

[10] A. H. Wickens, "The dynamic stability of railway vehicle wheelsets and bogies having profiled wheels," International Journal of Solids and Structures, vol. 1, no. 3, pp. 319-341, 1965.

[11] A. H. Wickens, Fundamentals of Rail Vehicles Dynamics: Guidance and Stability, Swets \& Zeitlinger, 2003.

[12] C. Knudsen, R. Feldberg, and A. Jaschinski, "Non-linear dynamic phenomena in the behaviour of a railway wheelset model," Nonlinear Dynamics, vol. 2, no. 5, pp. 389-404, 1991.

[13] M. Ahmadian and S. P. Yang, "Effect of system nonlinearities on locomotive bogie hunting stability," Vehicle System Dynamics, vol. 29, no. 6, pp. 365-384, 1998.
[14] M. Ahmadian and S. P. Yang, "Hopf bifurcation and hunting behavior in a rail wheelset with flange contact," Nonlinear Dynamics, vol. 15, no. 1, pp. 15-30, 1998.

[15] W. Q. Zhu, Nonlinear Stochastic Dynamics and Control, vol. 2, Science Press, Beijing, China, 2003.

[16] W. W. Liu, H. Y. Dai, and J. Zeng, "Research on stochastic stability of elastic constraint wheelset system," China Mechanical Engineering, vol. 24, no. 6, pp. 799-804, 2013 (Chinese).

[17] H. M. Sedighi and K. H. Shirazi, "Bifurcation analysis in hunting dynamical behavior in a railway bogie: using novel exact equivalent functions for discontinuous nonlinearities," Scientia Iranica B, vol. 19, no. 6, pp. 1493-1501, 2012.

[18] H. M. Sedighi and K. H. Shirazi, "A survey of hopf bifurcation analysis in nonlinear railway wheelset dynamics," Journal of Vibroengineering, vol. 14, no. 1, pp. 344-351, 2012.

[19] Y. He, H. Elmaraghy, and W. Elmaraghy, "A design analysis approach for improving the stability of dynamic systems with application to the design of car-trailer systems," Journal of Vibration and Control, vol. 11, no. 12, pp. 1487-1509, 2005.

[20] E. H. Law and R. S. Brand, "Analysis of the nonlinear dynamics of a railway vehicle wheelset," Journal of Dynamic Systems, Measurement and Control, vol. 95, no. 1, pp. 28-35, 1973.

[21] O. N. Kirillov, "Gyroscopic stabilization of non-conservative systems," Physics Letters A: General, Atomic and Solid State Physics, vol. 359, no. 3, pp. 204-210, 2006.

[22] L. Junfeng and W. Zhaolin, "Stability of non-conservative linear gyroscopic systems," Applied Mathematics and Mechanics, vol. 17, no. 12, pp. 1171-1175, 1996.

[23] http://www.umich.edu/ arclab/max/media/gyrovehicle.pdf.

[24] C. Pommer and W. Kliem, "Recent results on the stability and response bounds of linear systems: a review," Shock and Vibration Digest, vol. 38, no. 6, pp. 489-496, 2006.

[25] O. N. Kirillov, "Gyroscopic stabilization in the presence of nonconservative forces," Doklady Mathematics, vol. 76, no. 2, pp. 780-785, 2007.

[26] O. N. Kirillov, Nonconservative Stability Problems of Modern Physics, De Gruyter, Berlin, Germany.

[27] I. I. Metelitsyn, "The problem of gyroscopic stabilization," Doklady Akademii Nauk SSSR, vol. 86, no. 1, pp. 31-34, 1952.

[28] A. P. Seyranian and A. A. Mailybarv, Multiparameter Stability Theory with Mechanical Applications, World Scientific Publishing Company, Moscow, Russia, 2003.

[29] W. Kliem and A. P. Seyranian, "Metelitsyn's inequality and stability criteria for mechanical systems," Journal of Applied Mathematics and Mechanics, vol. 68, no. 2, pp. 199-205, 2004.

[30] D. V. Balandin, "On maximum energy of mechanical systems under shock disturbances," Shock and Vibration, vol. 1, no. 2, pp. 135-144, 1993.

[31] P. Lancaster and M. Tismenetsky, The Theory of Matrices, Academic Press, Orlando, Fla, USA, 2nd edition, 1985.

[32] A. H. Wickens, Fundamentals of Rail Vehicle Dynamics: Guidance and Stability, Swets \& Zeitlinger, 2003.

[33] H. Luo and Y. Wang, "Nonlinear vibration of a continuum rotor with transverse electromagnetic and bearing excitations," Shock and Vibration, vol. 19, no. 6, pp. 1297-1314, 2012.

[34] W. H. Zhang, Dynamic Simulation for Vehicles, China Railway Press, Beijing, China, 2006 (Chinese). 

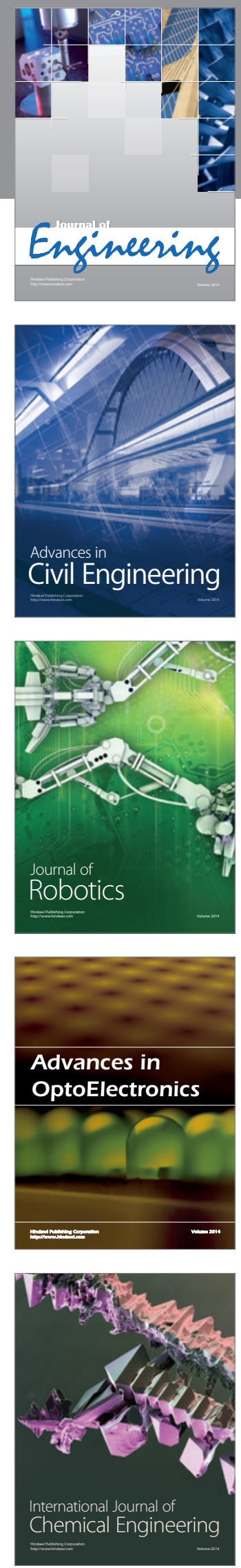

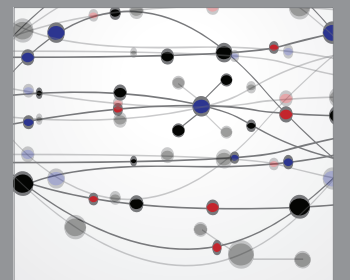

The Scientific World Journal
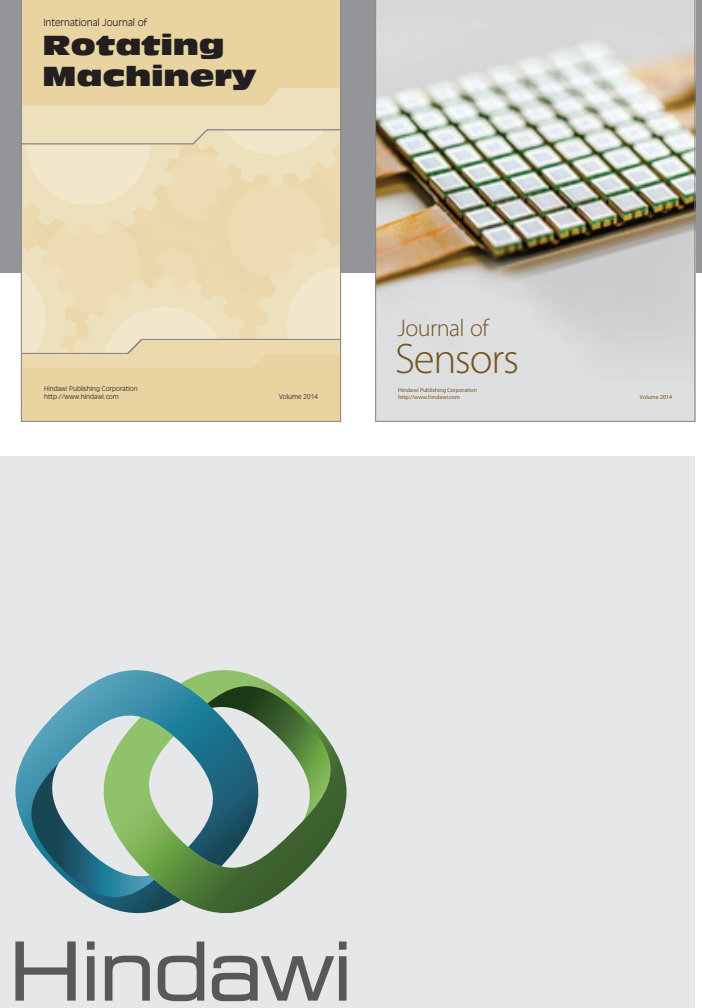

Submit your manuscripts at http://www.hindawi.com
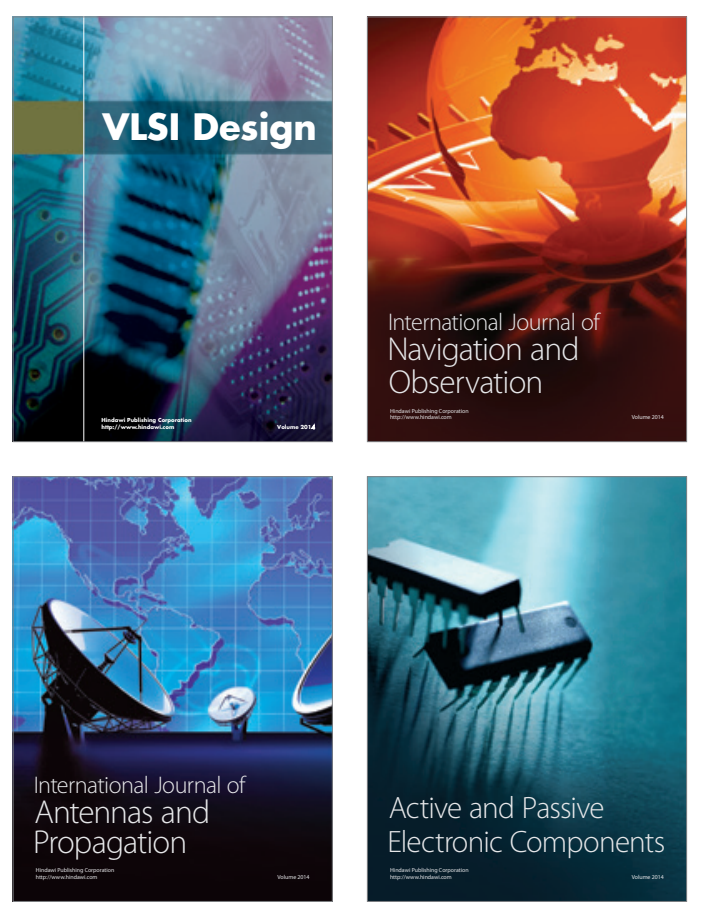
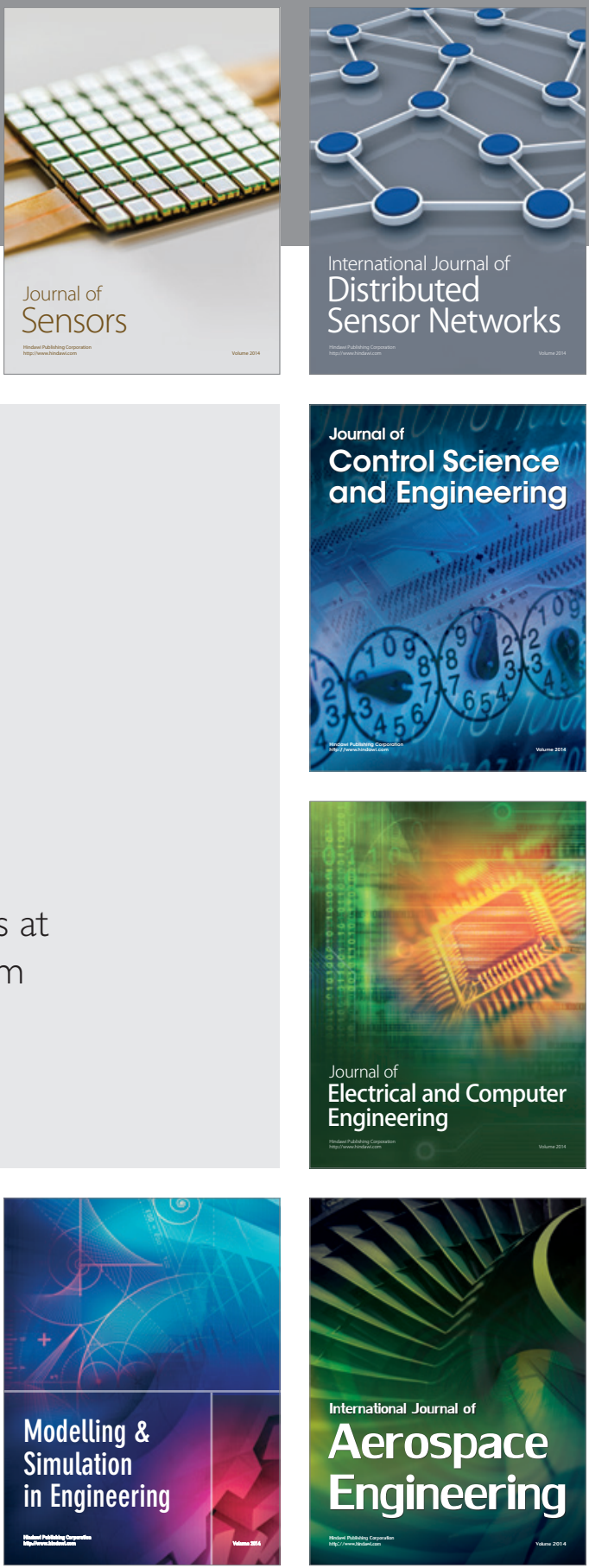

Journal of

Control Science

and Engineering
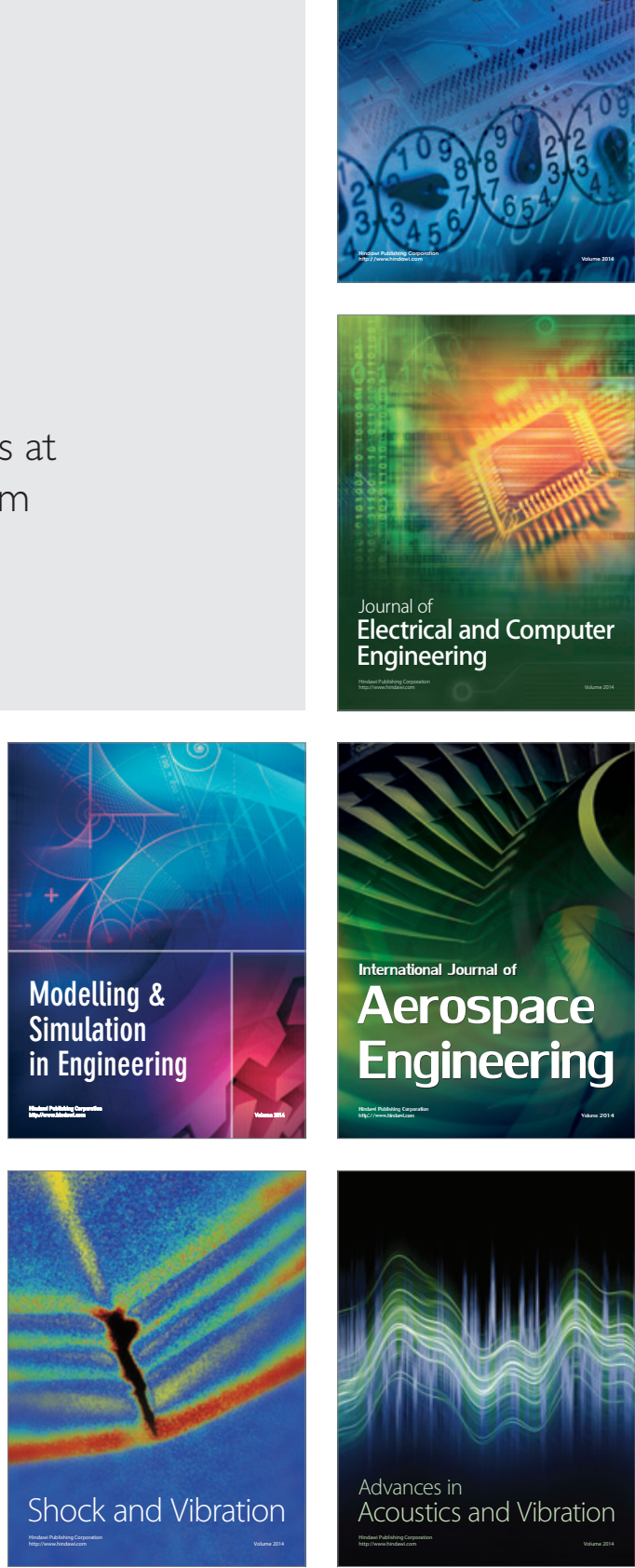